\title{
Mensch - Umwelt - Klima. Perspektiven der Quartärforschung in der Schweiz Einführung zum Themenheft
}

\section{Max Maisch, Zürich, Frank Preusser, Bern}

Das Quartär (Eiszeitalter) als jüngste Periode der Erdgeschichte umfasst die letzten 2,6 Mio. Jahre und dauert bis heute an. Diese Epoche ist durch markante Klimaschwankungen geprägt, die sowohl in geologischen Ablagerungen auf den Kontinenten und in den Ozeanen als auch in polaren Eisbohrkernen überliefert sind. Dem Bild einer nahezu vollständig vergletscherten Schweiz vor über 20'000 Jahren steht heute das Klimaszenario eines möglicherweise bald einmal völlig eisfreien Alpenraums gegenüber.

Im Verlauf des Quartärs tritt in Europa erstmals der Mensch auf. Seit rund 5'500 Jahren, dem Beginn der Sesshaftigkeit, ist der moderne Mensch (Homo sapiens) selber zu einem wichtigen Faktor im Umweltgefüge geworden. Er ist hauptverantwortlich für die Umwandlung der einstigen Natur- in die Kulturlandschaft heutiger Ausprägung. In den Warm- und Kaltphasen des Eiszeitalters entwickelten sich spezifische Pflanzen- und Tiervergesellschaftungen, anhand deren Überreste wichtige Aussagen über den Naturraum und die Lebens- und Wirtschaftsweise der Menschen in früheren Zeiten gemacht werden können. Stets und unzertrennbar mit der Quartärforschung verbunden ist der Aspekt des Klimas bzw. des Klimwandels in den unterschiedlichsten Raum- und Zeitmassstäben. Angesichts der gegenwärtig beschleunigten Entwicklung im «Treibhaus Erde» ist die Entschlüsselung der Klimageschichte zu einem der wichtigsten Sektoren quartärer «Vergangenheits-Forschung» geworden.

\section{Die Quartärforschung}

Seit den Zeiten von Louss Agassiz, einem der Hauptbegründer der Eiszeittheorie zu Beginn des 19. Jahrhunderts, kann die von den Eiszeitgletschern mehrfach überprägte Schweiz zweifellos als «Wiege der Quartärforschung» bezeichnet werden. Nach wie vor bietet sie für die verschiedensten Fragestellungen der Geo- und Humanwissenschaften einen aussergewöhnlichen Reichtum an Formationen und Fundstellen zur Entschlüsselung der Umwelt- und Menschheitsgeschichte. Eine ganze Reihe wissenschaftlicher Disziplinen beschäftigt sich heute mit dem Quartär und der Entwicklung des frühen Menschen. Kommunikation, Austausch und Vernetzung zwischen den oft unabhängig und isoliert forschenden Einzelpersonen oder
Gruppen aus Hochschulen, Instituten, Ämtern oder auch praxisnahen Unternehmen (z.B. Geologische Büros) sind zu zentralen, unabdingbaren Anliegen moderner Quartärforschung geworden.

\section{Übersicht über die Heftbeiträge}

Wesentliche Aspekte zur Theorie des Eiszeitalters wurden im Gebiet der Alpen entwickelt. Ein wichtiger Meilenstein war die von Penck und BrǘcKner (1901/09) eingeführte Untergliederung des Eiszeitalters in vier Eiszeiten (Günz, Mindel, Riss und Würm). Markus Fiebig und Frank Preusser resümieren in ihrem Artikel die Pionierzeit der Eiszeitenforschung und fassen den heutigen Kenntnisstand über den Ablauf des alpinen Quartärs zusammen. Sie weisen aber auch auf Schwachpunkte der klassischen Eiszeitengliederung hin, die sich im letzten Jahrzehnt mit dem Aufkommen neuer Datierungsmethoden herauskristallisiert haben. Insgesamt kam es während des Quartärs sicher zu wesentlich mehr Eisvorstössen als in der bald 100-jährigen Stratigraphie von PENK und BRÜCKNER enthalten sind.

Im Artikel von Markus Leuenberger werden die Grundlagen zur Rekonstruktion quartärer Klimaschwankungen anhand grönländischer Eisbohrkerne erläutert. Mittels verschiedener Isotope im Eis konnte hierbei gezeigt werden, dass es während der letzten Eiszeit über Grönland zu Temperaturschwankungen in der Grössenordnung von bis zu $10-15^{\circ} \mathrm{C}$ innerhalb weniger Jahrzehnte gekommen ist.

Brigitta Ammann et al. erläutern am Beispiel des Spätglazials, wie Organismen auf sich rapide ändernde Klimabedingungen reagiert haben. Evolutionäre Anpassung, Migration oder im Extremfall das Aussterben ganzer Arten sind die drei Möglichkeiten. Im Artikel werden die Migration bzw. Änderungen in der Populationsdynamik genauer analysiert. Es wird gezeigt, dass terrestrische und aquatische Lebensformen synchron auf Änderungen in den Umweltbedingungen reagiert haben.

Quartärforschung widmet sich direkt oder indirekt auch immer wieder der Frage nach der Herkunft, Entwicklung und Ausbreitung des Menschen in den verschiedenen Kulturabschnitten während des Eiszeitalters. Der Archäologe Pierre Crotti zeigt, wie die letzteiszeitlichen Eisvorstösse in den verschie- 
denen Höhenstufen eine entscheidende Rolle für die jeweiligen Lebensräume spielten. Unmittelbar davon betroffen sind die Fundstellen. Ihre Qualität und Unversehrtheit sind ganz entscheidend durch die Ausdehnung und Erosionswirkung der verschiedenen Vergletscherungsphasen beinflusst worden.

Ein wichtiger Zweig innerhalb der Quartärforschung beschäftigt sich mit der Datierung eiszeitlicher Bildungen und archäologischer Funde. Nur mittels einer verlässlichen Altersbestimmung lassen sich lokale Befunde in einen regionalen oder gar globalen Kontext stellen. IRKA HAJDAs et al. liefern einen generellen Überblick über das Potential der gebräuchlichsten Datierungsmethoden, die im Rahmen der Quartärforschung auf den Kontinenten eingesetzt werden. Es werden sowohl die Grundlagen kurz erläutert als auch die Probleme und das Potential diskutiert.

Der Beitrag von Luc BraillaRd und Michel MaUVILLY zur Morphogenese der Saaneschlucht steht beispielhaft dafür, wie geomorphologische und landschaftsgeschichtliche Fragestellungen erfolgsversprechend mit den Befunden, insbesondere den Datierungen aus archäologischen Grabungen, kombiniert werden können. Aus diesen zwei Betrachtungswinkeln eröffnen sich neue, unvorhergesehene Aspekte zu Alter und Dynamik von vermuteten Erosionsphasen, die bei einer isolierten Analyse verborgen geblieben wären.

Im Beitrag von Michel Blant, Marco Moretti und WILLY TINNER wird anhand von Knochenfunden typischer Fledermausarten mit unterschiedlichen UmweltAnsprüchen ein Zusammenhang mit der Vegetationsentwicklung im Holozän postuliert. Das Auftreten und Verschwinden bestimmter wärme- oder kälteliebender Arten widerspiegelt dabei typische Abfolgen, welche nur über klimagesteuerte Mechanismen zusammenspielen und erklärt werden können.

$\mathrm{Zu}$ durchaus regen Diskussionen dürfte der Beitrag von Peter Zwahlen zur Geologischen Karte des Prättigaus führen. Bei jahrelangen Kartierungsarbeiten, neueren Bohrungen und aktuellen Tunnelbauten sind im Schutt der Talhänge Holzfunde mit überraschenden Alterswerten zutage gefördert worden. Diese stellen die bisherige, scheinbar etablierte Gliederung der Spätglazialstadien auf den ersten Blick in Frage. Die augenfälligen Diskrepanzen zwingen jedoch alle am
Thema des alpinen Spätglazials Interessierten zu einer kritischen Überprüfung und Hinterfragung sowohl der geologischen Befundsituationen im Gelände als auch der Datierung von nicht «in situ» verwurzelt aufgefundenen, womöglich nachträglich umgelagerten Holzresten.

Die Beiträge in diesem Themenheft sind aus einer am 27. Oktober 2007 an der Universität Bern durchgeführten Fachtagung zur Quartärforschung in der Schweiz hervorgegangen. Die thematische Vielfalt dieses Heftes macht deutlich, dass eine ganze Palette von zentralen alten, wie aber auch neuen Themen der Quartärforschung mit durchaus überraschenden Ergebnissen angeschnitten wird. Damit werden hoffentlich fruchtbare Diskussionen angeregt. Dies ist auch der Zweck der an dieser Tagung neu gegründeten Schweizerischen Gesellschaft für Quartärforschung (CH-QUAT; http://ch-quat.ch).

\section{Ausblick}

Es ist zu hoffen, dass das vorliegende Themenheft Grundlage und Massstab zu aktuellen wie auch Ausgangspunkt und Motivation für künftige ForschungsKooperationen zwischen und jenseits der eigenen Disziplinengrenzen im Quartär bildet. In diesem Sinne ist es momentanes Abbild und sichtbares Artefakt aktiven quartären Schaffens mit zukunftsgerichteter Perspektive.

\section{Dank}

Die Autoren danken der swisstopo sowie der Schweizerischen Akademie der Naturwissenschaften (SCNAT) für die grosszügige finanzielle Unterstützung der Berner Tagung und dieses Sonderheftes.

Prof. Dr. Max Maisch, Geographisches Institut der Universität Zürich-Irchel, Gruppe für Glaziologie, Geomorphodynamik und Geochronologie, Winterthurertrasse 190, CH-8057 Zürich.

e-mail: max.maisch@geo.uzh.ch

PD Dr. Frank Preusser, Institut für Geologie der Universität Bern, Gruppe für Quartär- und Umweltgeologie, Baltzerstrasse 1+3, CH-3012 Bern.

e-mail: preusser@geo.unibe.ch 